\title{
Visibility science operations with the Keck Interferometer
}

Peter L. Wizinowich, Rachel L. Akeson, M. Mark Colavita, John Gathright, Eric Appleby, et al.

Peter L. Wizinowich, Rachel L. Akeson, M. Mark Colavita, John Gathright, Eric Appleby, James Bell, Andrew J. Booth, Wayne Dahl, Patricia Goude, Michael A. Hrynevych, Ian Lynn, Rafael Millan-Gabet, Christpher R. Neyman, Andy C. Rudeen, Tim Saloga, Kellee Summers, Kevin Tsubota, "Visibility science operations with the Keck Interferometer," Proc. SPIE 5491, New Frontiers in Stellar Interferometry, (20 October 2004); doi:

$10.1117 / 12.552746$

Event: SPIE Astronomical Telescopes + Instrumentation, 2004, Glasgow, United Kingdom 


\title{
Visibility science operations with the Keck Interferometer
}

\author{
Peter Wizinowich ${ }^{* a}$, Rachel Akeson ${ }^{\mathrm{b}}$, Mark Colavita ${ }^{\mathrm{c}}$, John Gathright ${ }^{\mathrm{a}}$, Eric Appleby ${ }^{\mathrm{a}}$, Jim Bell ${ }^{\mathrm{a}}$, \\ Andrew Booth ${ }^{\mathrm{c}}$, Wayne Dahl ${ }^{\mathrm{a}}$, Patricia Goude ${ }^{\mathrm{a}}$, Michael Hrynevych ${ }^{\mathrm{a}}$, Ian Lynn ${ }^{\mathrm{a}}$, Rafael Millan- \\ Gabet $^{\mathrm{b}}$, Chris Neyman ${ }^{\mathrm{a}}$, Andy Rudeen ${ }^{\mathrm{a}}$, Tim Saloga ${ }^{\mathrm{a}}$, Kellee Summers ${ }^{\mathrm{a}}$, Kevin Tsubota ${ }^{\mathrm{a}}$, \\ ${ }^{a}$ W. M. Keck Observatory, 65-1120 Mamalahoa Hwy, Kamuela, HI, USA 96743 \\ ${ }^{b}$ Michelson Science Center, California Institute of Technology, 770 South Wilson Avenue, \\ Pasadena, CA, USA 91125 \\ 'Jet Propulsion Laboratory, California Institute of Technology, 4800 Oak Grove Drive, \\ Pasadena, CA, USA 91109
}

\begin{abstract}
The visibility science mode of the Keck Interferometer fully transitioned into operations with the successful completion of its operational readiness review in April 2004. The goal of this paper is to describe this science mode and the operations structure that supports it.
\end{abstract}

Keywords: Interferometer, operations, visibility, Keck

\section{INTRODUCTION}

First fringes between the two Keck telescopes were achieved in March 2001. Subsequent to first light, approximately 22 Keck-Keck engineering nights per year have been scheduled, divided into 8 observing runs per year. During this period the instrument has transitioned from its engineering phase to operations. A major operations milestone along this path was the handover of responsibility for operating the interferometer for shared-risk visibility science in April 2003, from the Jet Propulsion Laboratory (JPL) to the W. M. Keck Observatory (WMKO) and Michelson Science Center (MSC).

Another major milestone was the publication of the first two science papers from the Keck Interferometer in the summer of 2003. The National Aeronautics and Space Administration (NASA) had selected four shared-risk science teams in 2001 to participate with the internal development/science team in the demonstration of first science with the Keck Interferometer. Beginning in June 2002, each observing run has had at least a half night of scheduled shared-risk science observing. This transitioned in 2003 to more than half the observing nights being used for shared-risk science. In addition, Caltech and NASA time allocation committees have approved time for shared-risk interferometry observations. The visibility science results from the NASA-selected shared-risk science teams and the internal science team are described in other papers at this conference. ${ }^{1,2}$

The most recent milestone has been the successful completion of the Visibility science mode Operational Readiness Review (V ${ }^{2}$ ORR) in April 2004. This marks the formal transition of this mode from development to operations, and the release of this science mode for general science usage. The organizations responsible for operations include the WMKO for operating the interferometer and the MSC for pre- and post-observing support.

\section{INSTRUMENT OVERVIEW}

The instrument has been described elsewhere. ${ }^{3,4}$ The light is collected by the two Keck $10-\mathrm{m}$ telescopes separated by 85 $\mathrm{m}$. The wavefronts are corrected by high-order adaptive optics (AO) systems located on the Nasmyth platform of each telescope. ${ }^{5}$ A 112-mm diameter collimated beam from each telescope, corresponding to a 9-m inscribed circle on the Keck primary mirror, is sent, via the dual star module (DSM), coude and beam transport mirrors, to the Long Delay

* peterw@keck.hawaii.edu; phone 808-885-7887; fax 808-881-3535; http://www.keckobservatory.org; W. M. Keck Observatory, 651120 Mamalahoa Hwy, Kamuela, HI 96743 
Lines (LDL) and then the Fast Delay Lines (FDL). Figure 1 shows the optical path from one of the two telescopes through the delay lines to the beam-combiner lab. The dome and basement environments are separated by a fused silica window after the first beam transport mirror. The LDLs are stationary during an observation; observing programs are currently designed so that at most four LDL positions are required during the night. The FDL carts are multi-stage servo systems used to track the fringes based on a combination of inputs including sidereal motion, FDL-internal and beam train (fringe tracker to DSM) piston metrology systems, telescope accelerometer data, and fringe tracker camera data. After the FDLs the beam is compressed by a factor of 4 and sent into the beam-combining lab. The H- and Kband light is sent to the beam combiner that feeds the fringe tracker camera via single-mode fibers. The J-band, and some H-band light, is sent to the angle tracker camera. The remaining visible light goes to an alignment camera. The angle tracker camera controls a fast tip/tilt mirror in the beam-combining lab to remove tip/tilt introduced after the AO system; this mirror is offloaded to the AO system tip/tilt mirror which is, in turn, offloaded to telescope pointing.

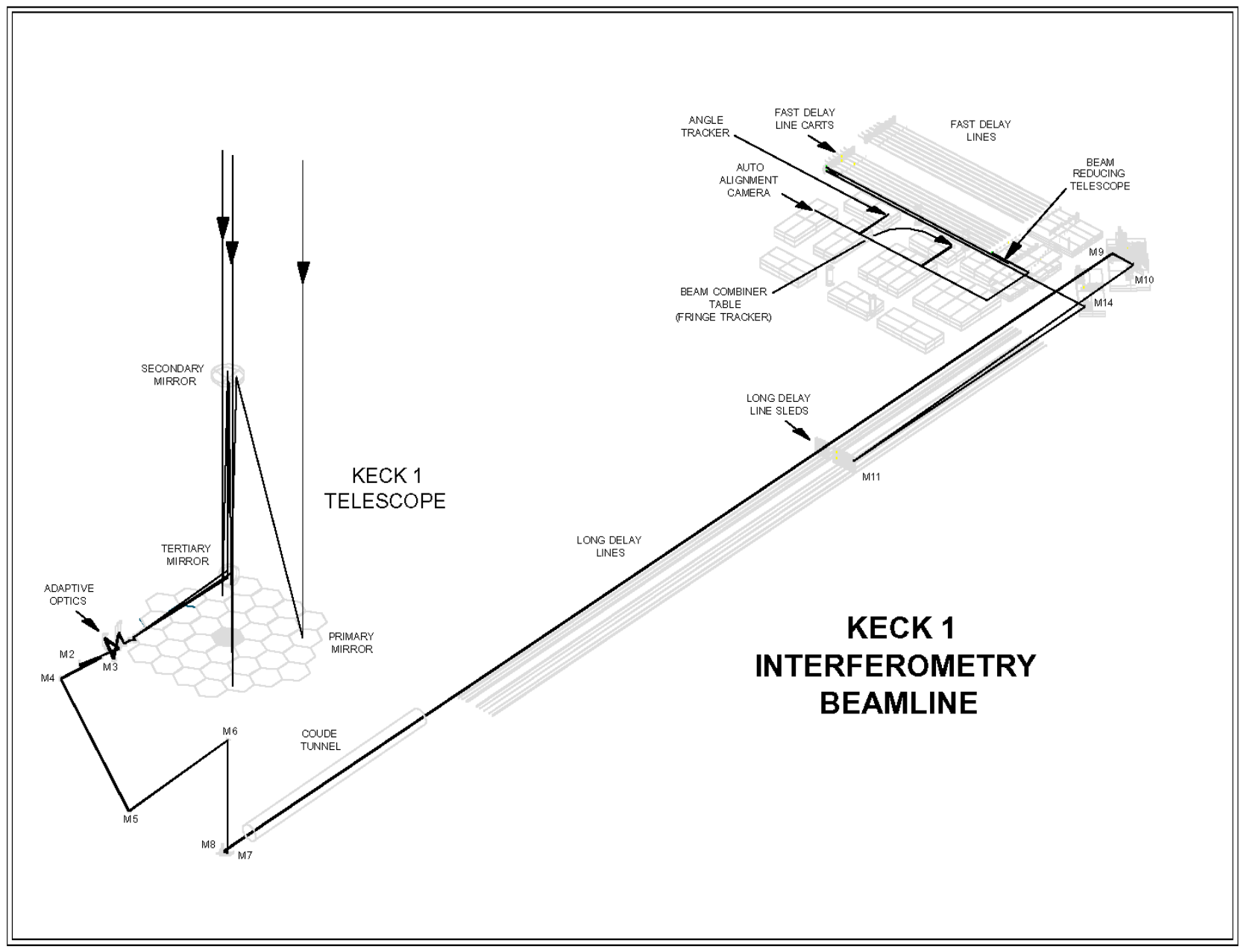

Fig. 1. Optical path from the Keck I telescope through the delay lines to the beam-combiner lab.

The Real-Time Control (RTC) system, developed at JPL, provides all high-rate control. All medium-rate control is provided by the twin Observatory standards of Experimental Physics and Industrial Control System (EPICS) and Keck Task Library (KTL) keywords. The physical hardware is based around VME crates with VxWorks and PowerPC boards. The GUIs (Java and Tcl/Tk) and other tools, like the configuration manager, archiver, sequencer and planning tool, run on UNIX machines. 


\section{OBSERVING SEQUENCE}

Figure 2 shows the timeline for a typical interferometer observation or scan. The columns represent activities carried out by the telescope, AO system, and interferometer, respectively. The steps in this sequence are described in more detail in the bullets following the figure.

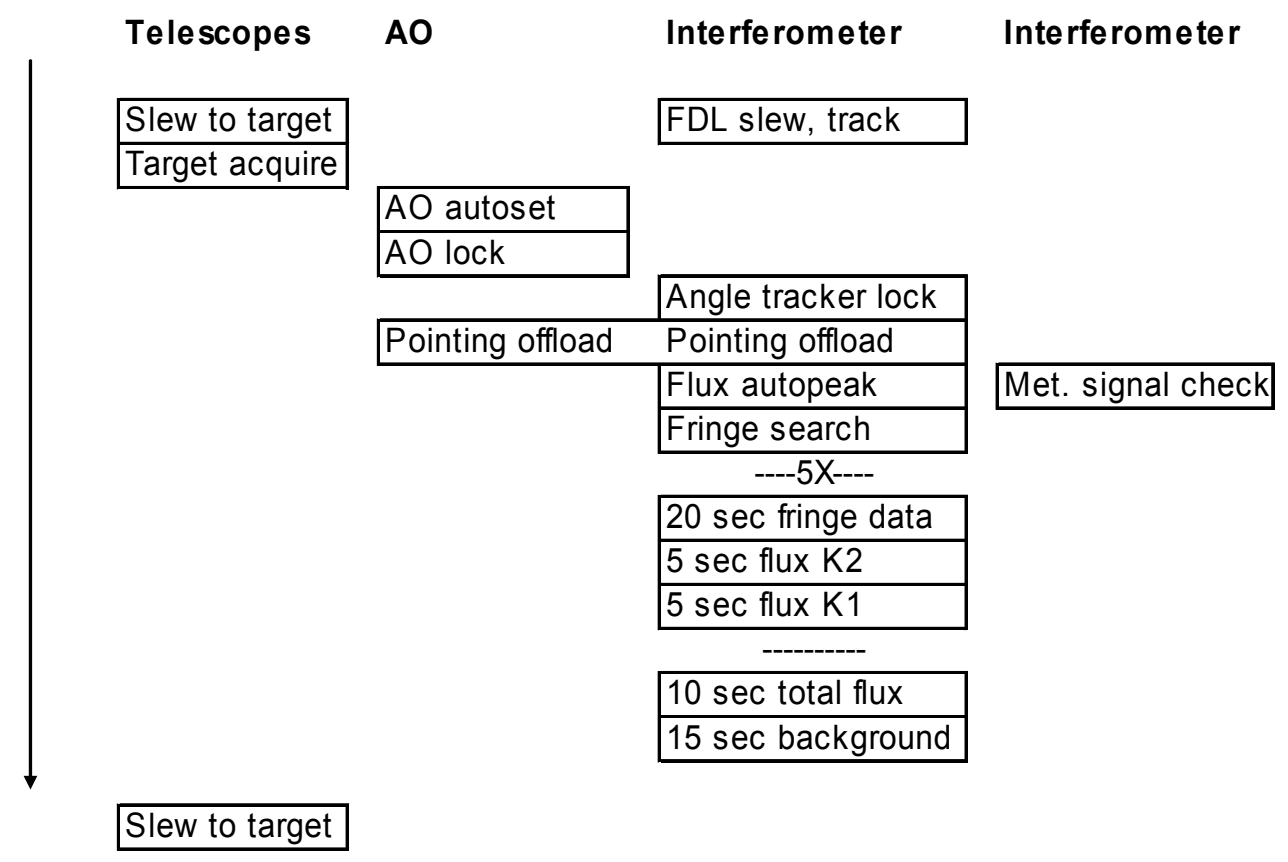

Fig. 2. Timeline for a typical observing scan.

- Slew to target. A new observation begins by slewing to the next target. The target is loaded by the interferometer operator from a target list. The target list includes coordinates and magnitudes. The telescope sequencer uses the target coordinates to automatically slew both telescopes.

- FDL slew, track. The interferometer sequencer uses the target coordinates to position the FDLs at the appropriate position to find fringes.

- Target acquire. When the slew is complete the telescope sequencer triggers the auto-acquisition tool to acquire the target. An image is taken using the AO acquisition camera, the brightest target in the field is identified and the target is centered at the appropriate location for $\mathrm{AO}$ acquisition.

- AO autoset \& lock. The telescope operator then triggers the AO autosetting tool. Based on the magnitude and color of the target, provided in the target list, the AO system sets the wavefront sensor frame rate and control loop gains, takes a sky image for background subtraction, and closes the AO loops. These values are automatically adjusted, in a single iteration, if the wavefront sensor intensity is outside of the optimal range. Once the AO loops are locked the average tip/tilt error on the tip/tilt mirror and the average focus on the deformable mirror are regularly offloaded to the telescope.

- Angle tracker lock. The interferometer operator then closes the angle tracker tip/tilt loop to center the target on the angle tracker camera.

- Pointing offload. The offset (pointing) of the angle tracker tip/tilt mirror is automatically sent to the AO system as a centroid offset. These centroid offsets are 1-shot offloaded to the AO field steering mirrors.

- The interferometer sequencer then automatically performs the following sequence:

- Flux autopeak. The flux from each telescope is individually peaked up on the fringe tracker by scanning a mirror feeding light into the single mode fiber. The mirror is left centered at the peak signal position. 
- Fringe search. The standard search is a raster search about the starting position until fringes are found.

- $20 \mathrm{sec}$ fringe data, $5 \mathrm{sec}$ flux K2, $5 \mathrm{sec}$ flux K1. This sequence is carried out 5 times. After taking 20 $\mathrm{sec}$ of fringe data, each of the telescope beams is shuttered for $5 \mathrm{sec}$ in order to measure the average flux from the other telescope.

- $10 \mathrm{sec}$ total flux, $15 \mathrm{sec}$ background. Both shutters are open off of the fringe position for $10 \mathrm{sec}$, followed by $15 \mathrm{sec}$ with both shutters closed.

- Metrology signal check. In parallel with the flux autopeak step above, the piston metrology signal (from the fringe tracker to a retro just before reaching the AO system) is checked and peaked up.

\section{VISIBILITY MEASUREMENT}

A single fringe measurement (typically $\sim 5 \mathrm{~ms}$ ) consists of four synchronized fringe tracker camera reads (a,b,c,d), and one reset $(\mathrm{z})$, as the FDL scans over one wavelength (typically $\sim 2 \mu \mathrm{m}$ ). A visibility measurement is basically $\mathrm{V}^{2}=$ $\left(\pi^{2} / 2\right)\left\{[(\mathrm{a}-\mathrm{z})-(\mathrm{c}-\mathrm{b})]^{2}+[(\mathrm{b}-\mathrm{a})-(\mathrm{d}-\mathrm{c})]^{2}\right\} /(\mathrm{d}-\mathrm{z})^{2}$, where the various reads have been corrected for biases (background, quadrature effect and photon counting), read noise, and scaling (dewarping). In addition, post-processing corrects for intensity imbalances, determines the average visibility, and corrects for the system visibility using calibrator stars. This last point is a significant driver for the observing sequences. Generally, calibrator stars are observed before and after each science target measurement, and at least two different calibrators are used per science target.

\section{PERFORMANCE}

NASA's requirements for the Keck Interferometer derive from the needs of NASA's Origins theme. Level 1 requirements are defined in a Program Level Requirements Appendix (PLRA) to the Navigator Program Plan. The PLRA defines the science objectives and the performance requirements necessary to achieve them. The PLRA contains science requirements for exo-zodiacal dust measurements at $10 \mu \mathrm{m}$ via nulling and for differential phase measurements at 2-5 $\mu \mathrm{m}$. It does not contain any science requirement for a stand-alone $\mathrm{V}^{2}$ capability. However, the performance levels that enable $\mathrm{V}^{2}$ must be achieved in order to meet the nulling and differential phase science requirements. $\mathrm{V}^{2}$ performance is, therefore, stated in terms of a "goal" in the PLRA.

The PLRA Level 1 performance goals/requirements for $\mathrm{V}^{2}$ included the following:

- Goal: $5 \%$ rms accuracy $\mathrm{V}^{2}$ amplitude measurements for targets as faint as $\mathrm{K}=9$ using the two Keck telescopes.

- Requirement (for all modes): Provide at least $90 \%$ uptime for the interferometer.

- Goal: Provide at least $80 \%$ uptime for the two 10-m telescopes and their AO systems.

A set of test binaries and calibrators was used to demonstrate the data accuracy requirement. The standard observing sequence and data reduction pipeline were used. Predictions were based on orbit solutions from Palomar Testbed Interferometer observations. The resultant data errors include statistical errors and systematic errors in the external calibration (such as the finite size of the calibrator stars). The result was an rms accuracy of approximately $1 \%$ for the experiments with $\mathrm{K}=5.9$ and 8.0 magnitude binaries, and $5.4 \%$ for a $\mathrm{K}=9.0$ magnitude binary. Figure 3 shows the results for the $\mathrm{K}=8.0$ case.

The interferometer and facility (telescope and AO systems) uptime was monitored during each observing run between the handover and operational readiness review. The results are displayed in Fig. 4. For the three runs immediately prior to the ORR the average interferometer uptime was $94 \%$ and the minimum was $89 \%$. For the facility, the average uptime was $88 \%$ and the minimum was $87 \%$. The uptime performance has met the requirement/goal. Moreover, this tracking metric proved useful in identifying problem areas, and prioritizing where to apply resources.

Level 2 performance requirements were generated by the project team to support maximizing the science at the specified performance level. Table 1 lists these requirements and the achieved performance. The first three items address overall efficiency, important to all science modes. The spectrometer configuration items are $\mathrm{V}^{2}$ specific. The reconfiguration guidelines are mostly relevant to $\mathrm{V}^{2}$, but also address overall efficiency. The "calibrations provided per star" is another $\mathrm{V}^{2}$ specific item, although similar approaches are needed for other modes. The target requirements address overall instrument sensitivity and sky coverage. A few details on the less obvious requirements are provided below. 

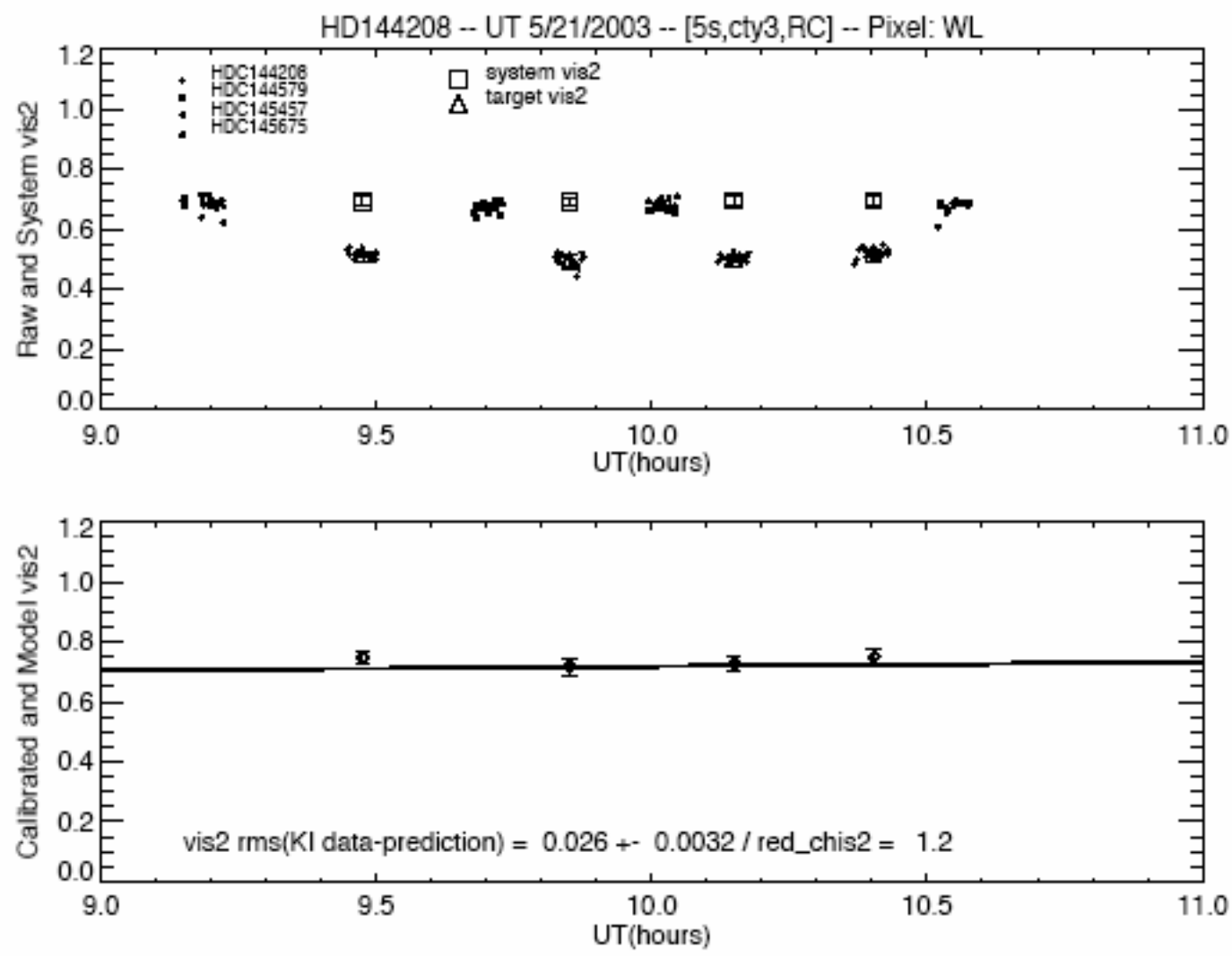

Fig. 3. $\mathrm{V}^{2}$ rms accuracy plots for a $\mathrm{K}=8.0$ binary.

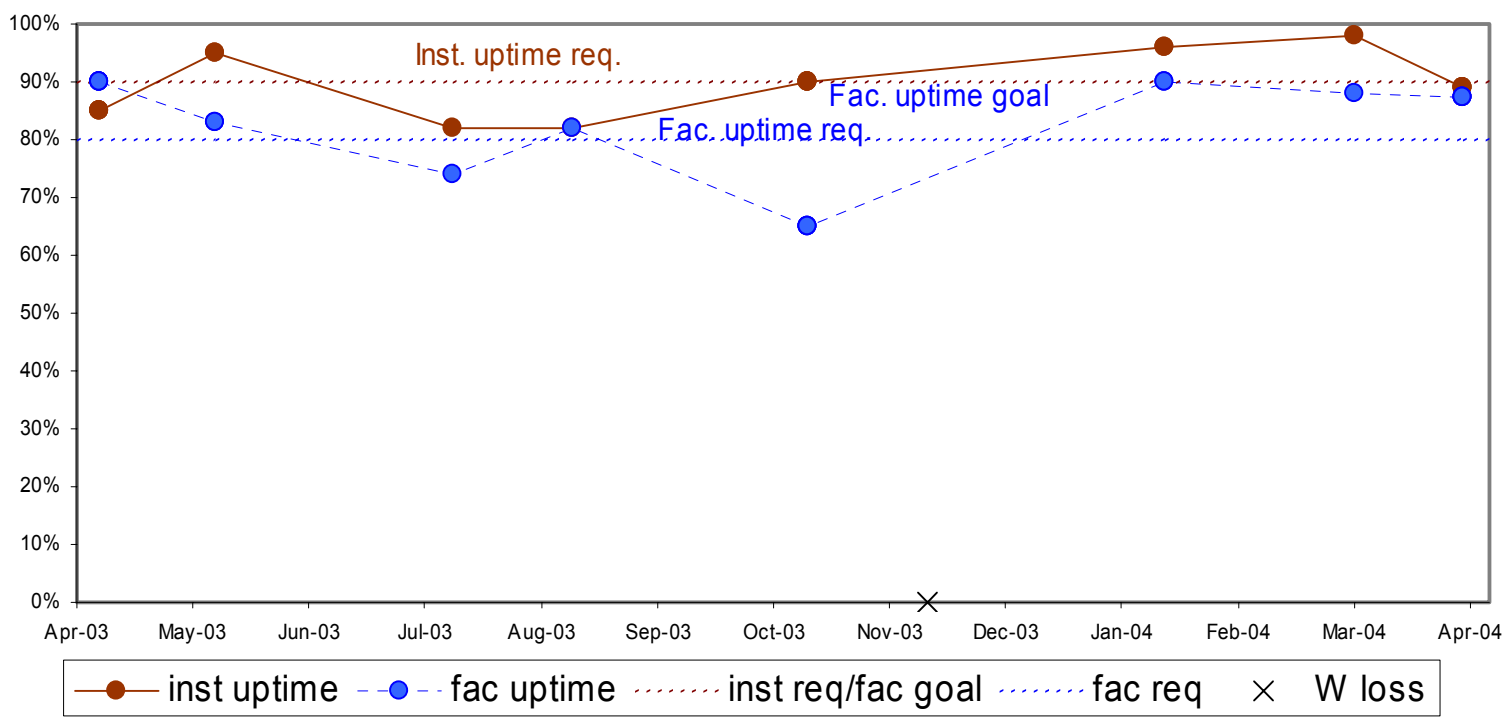

Fig. 4. System uptime history. 
Table 1. $\mathrm{V}^{2}$ ORR performance requirements and achieved status.

\begin{tabular}{|c|c|c|c|}
\hline & Item & Requirement & Status \\
\hline P2.1 & Scan rate & 6 scans $/ \mathrm{hr}$ & $\begin{array}{l}\text { Peak burst rate } \geq 6 \text {; Peak night, avg } \\
\text { rate } \geq 5.5 ; \text { Average } 4.2\end{array}$ \\
\hline P2.2 & LDL reconfiguration time & 10 min, automatic & $\begin{array}{l}10 \text { of last } 12 \text { moves: < } 10 \text { min } \\
\text { However, additional setup overhead } \\
\text { affects scan rate }\end{array}$ \\
\hline P2.3 & Camera mode/parameter reconfiguration time & $10 \mathrm{~min}$ & Yes \\
\hline- & Spectrometer configurations [y/n] & & \\
\hline P2.4 & WL: K, Spec: K4 & $y$ & Yes \\
\hline P2.5 & WL: K, Spec: K8 & $y$ & $\begin{array}{l}\text { Hardware in place, but not currently } \\
\text { implemented }\end{array}$ \\
\hline P2.6 & WL: H, Spec: H4 & $y$ & Yes \\
\hline- & Reconfiguration guidelines & & \\
\hline P2.7 & LDL & between sequences & Yes \\
\hline P2.8 & Camera modes/parameters & between sequences & Yes \\
\hline P2.9 & Calibrations provided per star & $\begin{array}{l}\text { K1 flux, K2 flux, } \\
\text { total flux, } \\
\text { background }\end{array}$ & Yes \\
\hline P2.10 & Additional nightly calibrations & $\begin{array}{l}\text { spectrometer } \\
\text { calibration filter flux }\end{array}$ & Yes, but with different approach \\
\hline- & Target requirements & & \\
\hline P2.11 & $\mathrm{R}$ band magnitude $(\mathrm{AO})$ & 11 & Yes \\
\hline P2.12 & Off-axis $A O$ reference allowed $[y / n]$ & y & Yes \\
\hline P2.13 & J band magnitude (angle tracker @ 100 Hz) & 8 & Yes \\
\hline P2.14 & J band magnitude (angle tracker @ 20 Hz) & 10 & Yes \\
\hline P2.15 & Minimum K-band $\mathrm{V}^{2}$ for tracking (bright sources) & 0.1 & Yes \\
\hline P2.16 & Zenith angle & $2.5-50 \mathrm{deg}$ & Yes \\
\hline
\end{tabular}

The first item in Table 1 is the scan rate. Figure 5 shows the $\mathrm{V}^{2}$ scans for a recent science night. The number of scans/hour is computed for science time only; hours that are excluded include down time, engineering time and observations of sources fainter than the standard limits. Figure 6 shows the scans/hour history for the past year. As can be seen from this plot we frequently achieve or exceed the 6 scans/hour requirement for periods of an observing run, however, the overall average rate is only $4.2 \mathrm{scans} /$ hour. The factors that have resulted in a lower than desired average rate include:

- Target factors such as low source magnitude (faint sources may require manual optimization and take longer to collect fringe data) or unknown source size (significant time can be spent in searching for fringes on too large a source).

- Efficiency factors such as overhead (i.e., LDL moves taking longer) and recovery from procedural errors or faults.

- Environmental factors such as poor seeing, cloud cover, wind shake, new vibrations or even recovery from power outages.

We intend to improve on this performance through the reduction in overhead and improvements in operational and fault recovery procedures. 


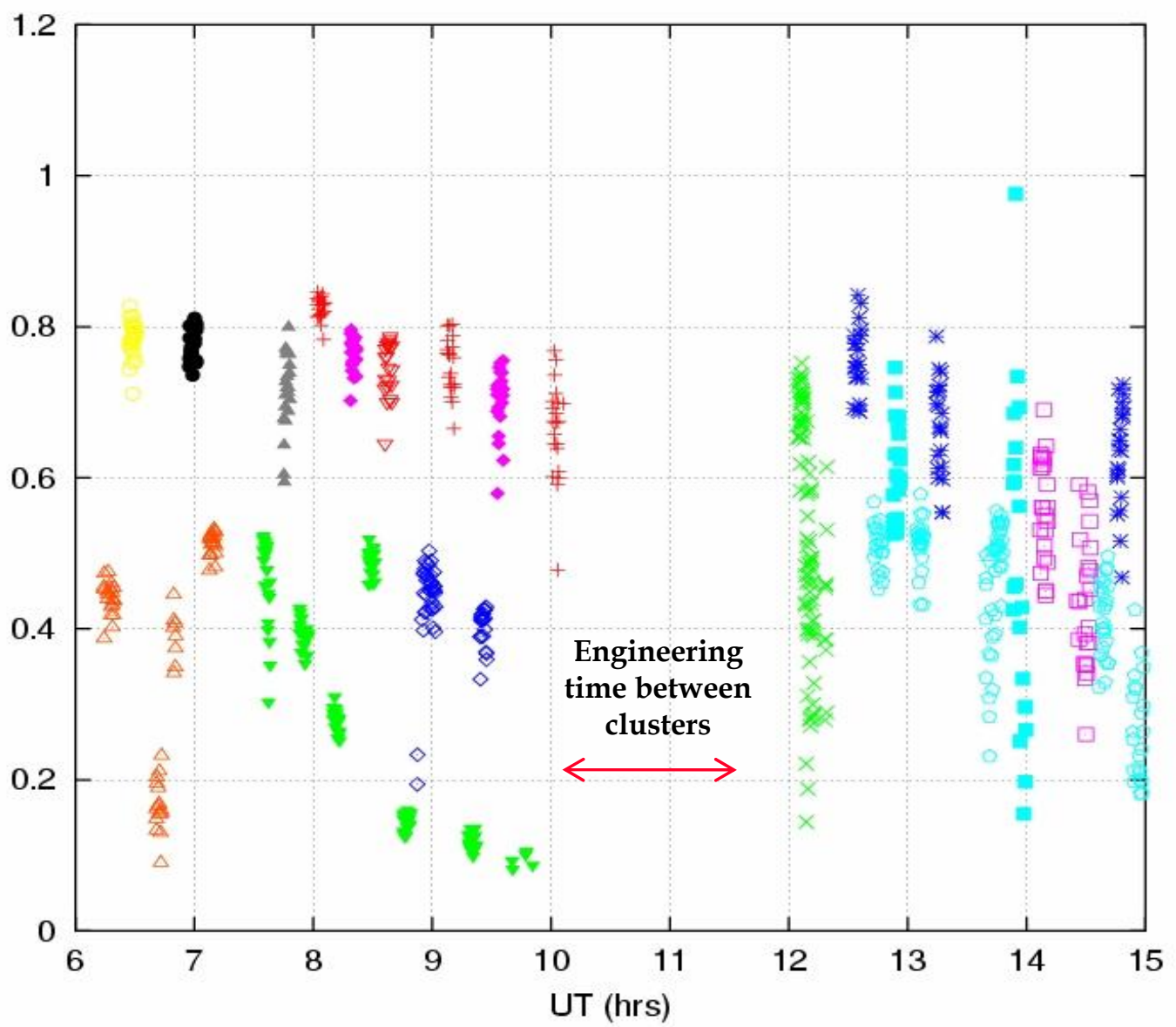

Fig. 5. Plot of $\mathrm{V}^{2}$ versus time, showing the scan rate on the night of April 5, 2004. Each cluster with the same symbol is a scan.

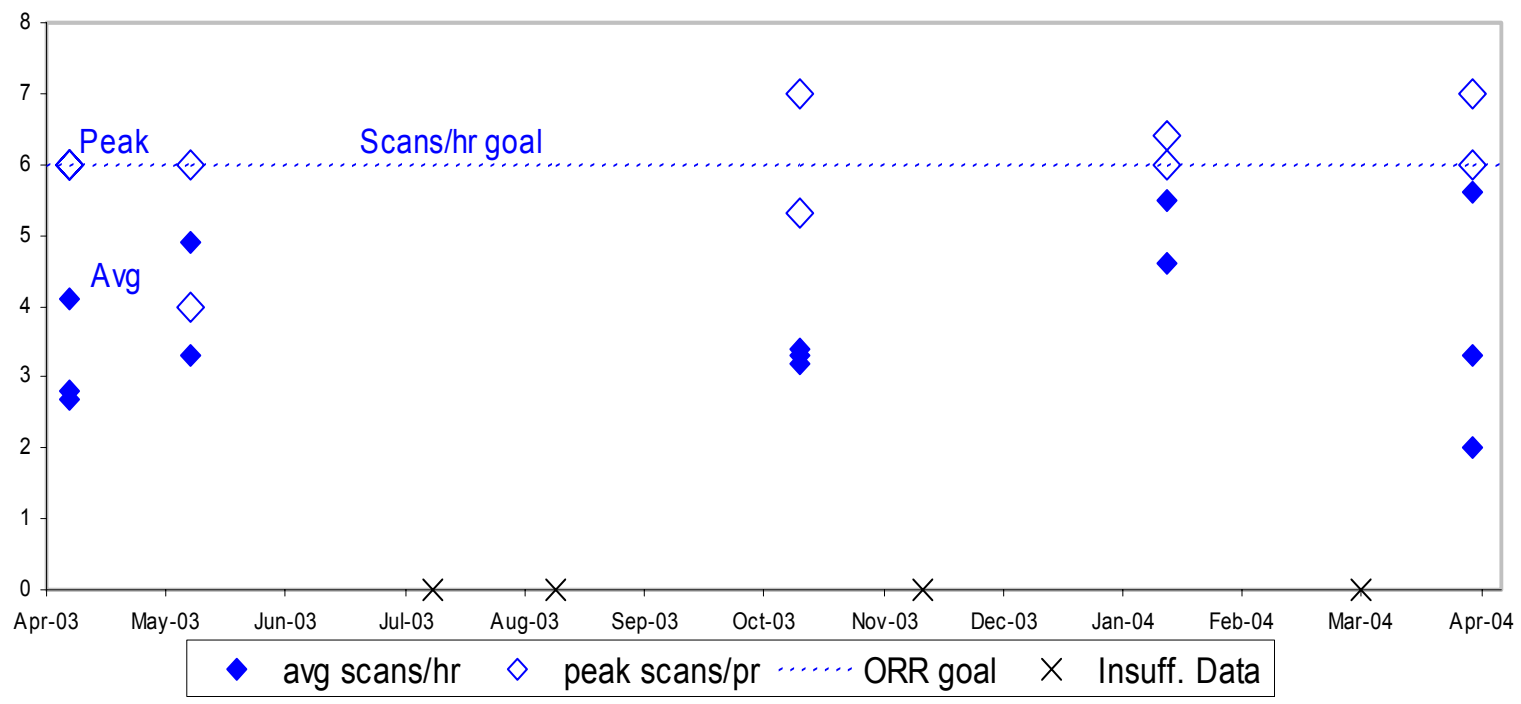

Fig. 6. Scans per hour history. 
The fast delay lines have a range of $\pm 15 \mathrm{~m}$. Greater sky coverage requires the movement of the LDLs. Realignments are required after each LDL move since flat mirrors are used. Another paper at this conference ${ }^{6}$ describes the autoalignment procedure. The LDL move and realignment has typically been accomplished in 10 minutes, per the goal, in the last 3 observing runs. However, the time between fringes is significantly longer and we therefore normally allot a total of 45 minutes in the observing plan for an LDL move. We are continuing to working on procedural improvements with the goal of $\sim 20$ minutes between fringes.

Figure 7 shows the currently advertised $\mathrm{V}^{2}$ science mode magnitude limits for the AO, Keck angle tracker (KAT) and Fringe Tracker (FT) systems. The overlaid plots of $\mathrm{R}=11$ magnitude A0 and K0 stars indicates that the AO system is the limiting factor for a K0 star, while both the KAT and FT are limiting factors for an A0 star. A few words are appropriate with respect to these magnitude limits. They are not fundamental limits and several options exist for improving them. However, these criteria were initially set to achieve NASA's nulling and differential phase science goals, and the priority on improving these limits is low compared to the development of the these future science modes.

More details on the Keck Interferometer's $\mathrm{V}^{2}$ science mode sensitivity and observing parameters can be found at $\mathrm{http}: / / \mathrm{msc} . c a l t e c h . e d u / K I S u p p o r t / \mathrm{v} 2 / \mathrm{v} 2$ sensitivity.html.

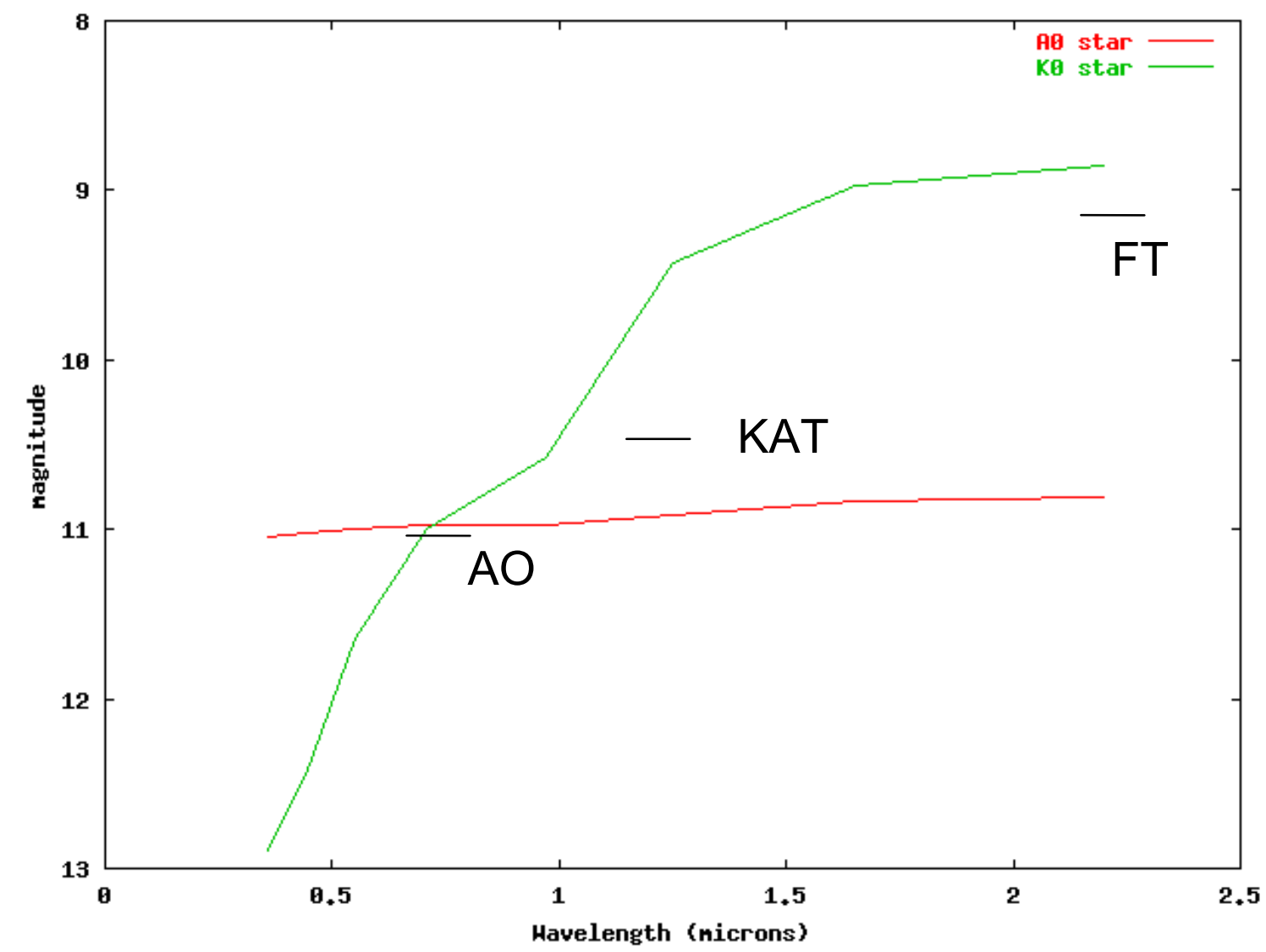

Fig. 7. Current limiting magnitudes for the adaptive optics system (AO) at R-band, the angle tracker (KAT) at J-band and the fringe tracker (FT) at K-band. The magnitude versus wavelength for $\mathrm{R}=11$ magnitude $\mathrm{A} 0$ and $\mathrm{K} 0$ stars is also shown.

\section{OPERATIONS MODEL}

The $\mathrm{V}^{2}$ science mode is the first of a series of Keck Interferometer science modes to be handed over to operations. The purpose of this section is to describe the operations model into which we have just begun to transition with the completion of the $\mathrm{V}^{2}$ ORR. 
Interferometer operations are managed by the WMKO and science operations are managed by the MSC. WMKO is responsible for the management, operation, maintenance, and upgrade of the Keck Interferometer facilities in Hawaii. The MSC provides Keck Interferometer science operations system operations and full lifecycle user support. WMKO executes the science plans provided by the MSC, supports the MSC's role, and maintains MSC tools in Hawaii. All observations are performed via service observing by WMKO personnel. Figure 8 schematically illustrates the $\mathrm{V}^{2}$ observing model from the users' perspective.

In addition to the performance requirements discussed above, the PLRA provided a set of operational requirements. The top-level PLRA guidance was to "Provide an operational infrastructure including trained staff, facilities, hardware and software, so that the Keck Interferometer will operate as a facility for use by the community scientists." The PLRA explicitly provided 10 level 1 requirements on the MSC from which 17 level 2 functional requirements were derived. Since WMKO is not a NASA organization, no WMKO-specific level 1 requirements were included in the PLRA. However, NASA did provide a number of guiding questions which were used to generate a set of 22 level 2 requirements for WMKO. Overall, we were able to demonstrate good compliance with these requirements at the $\mathrm{V}^{2}$ ORR.

Since we are still in the development phase for the other Keck Interferometer science modes, we have currently limited the number of $\mathrm{V}^{2}$ science nights to six nights per six-month semester. These have been shared-risk nights in 2004, but with the successful completion of the $\mathrm{V}^{2}$ ORR these will move to general science nights in 2005.

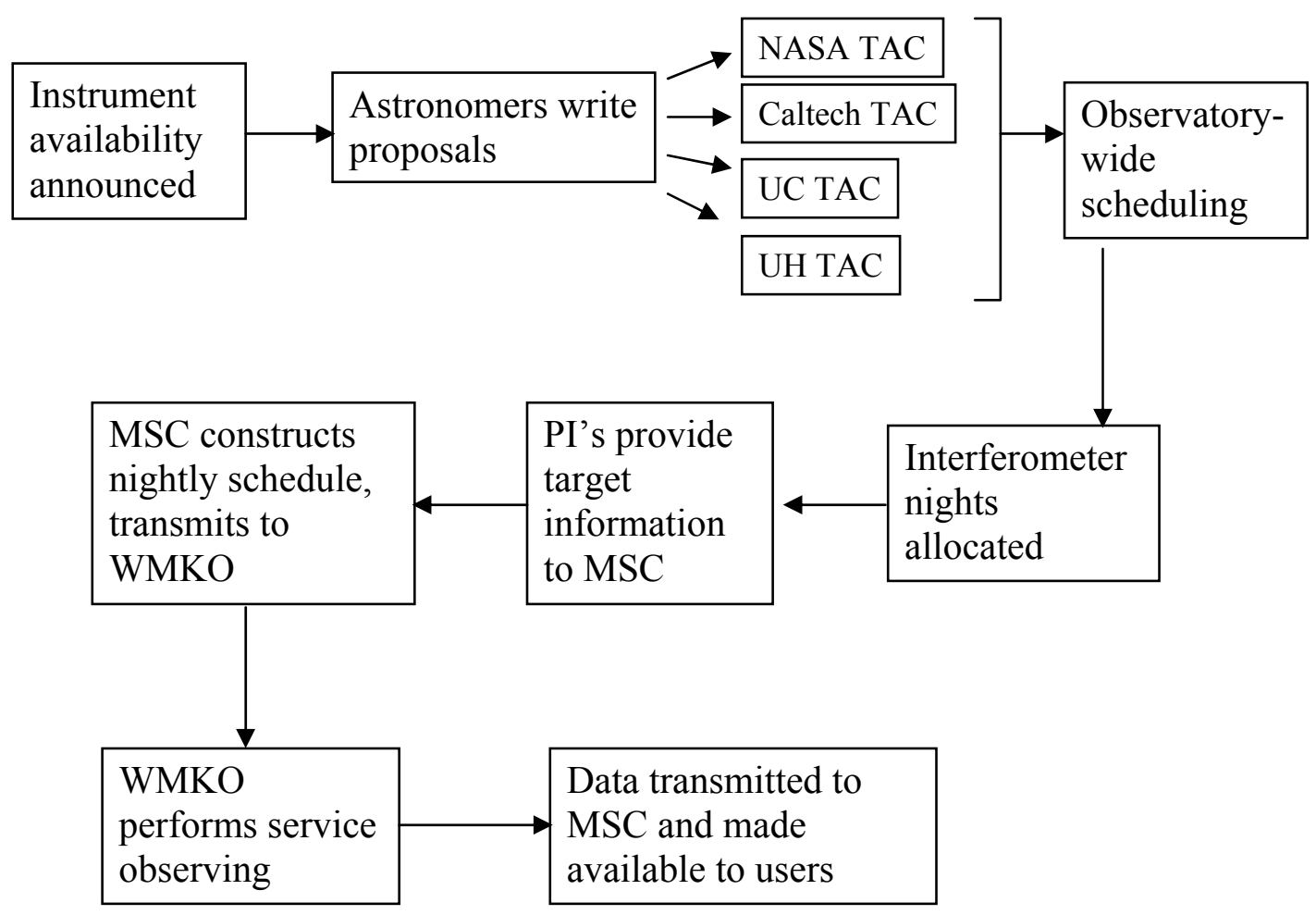

Fig. 8. Keck Interferometer observing from the users' perspective. 


\section{MSC OPERATIONS}

The MSC support of Keck Interferometer operations includes contact scientists, developers and pipeline operators. The science operations system includes:

1. Proposal preparation support

2. Proposal solicitation and selection administration

3. Observation planning support

4. Observation scheduling

5. Summit data infrastructure and real time visualizations

6. Data reduction and transmission pipeline

7. Data cataloguing, archiving and user access

8. Data quality assessment and instrument monitoring

9. Data calibration tools and contact science support

Those interested in further details can view the MSC's Keck Interferometer Support page at http://msc.caltech.edu/KISupport/index.html or contact the MSC at msc-help@ipac.caltech.edu.

\section{WMKO OPERATIONS}

The current core WMKO interferometer staff includes three interferometer specialists, three software engineers, one electronics engineer, one mechanical engineer, two technicians and a recently hired interferometer operator. These core personnel are involved in both operations and development. Other WMKO personnel participate in the development efforts and to a small extent in operations.

Figure 9 shows both the headquarters and summit interferometer control rooms. The summit control room is generally used to support hands-on work on the summit including pre-observing daytime summit checkout. Observations are performed from the headquarters control room. One of the interferometer specialists operates the interferometer while a second specialist runs the MSC tools and coordinates the carrying out of the science plan. They communicate with the Keck I and II telescope operators on the summit, and the summit interferometer control room via three separate video links. We still maintain at least one interferometer person on the summit during observing in case of problems (thankfully, this has become a boring job). A WMKO software engineer, an MSC person, and a JPL person are on-call.

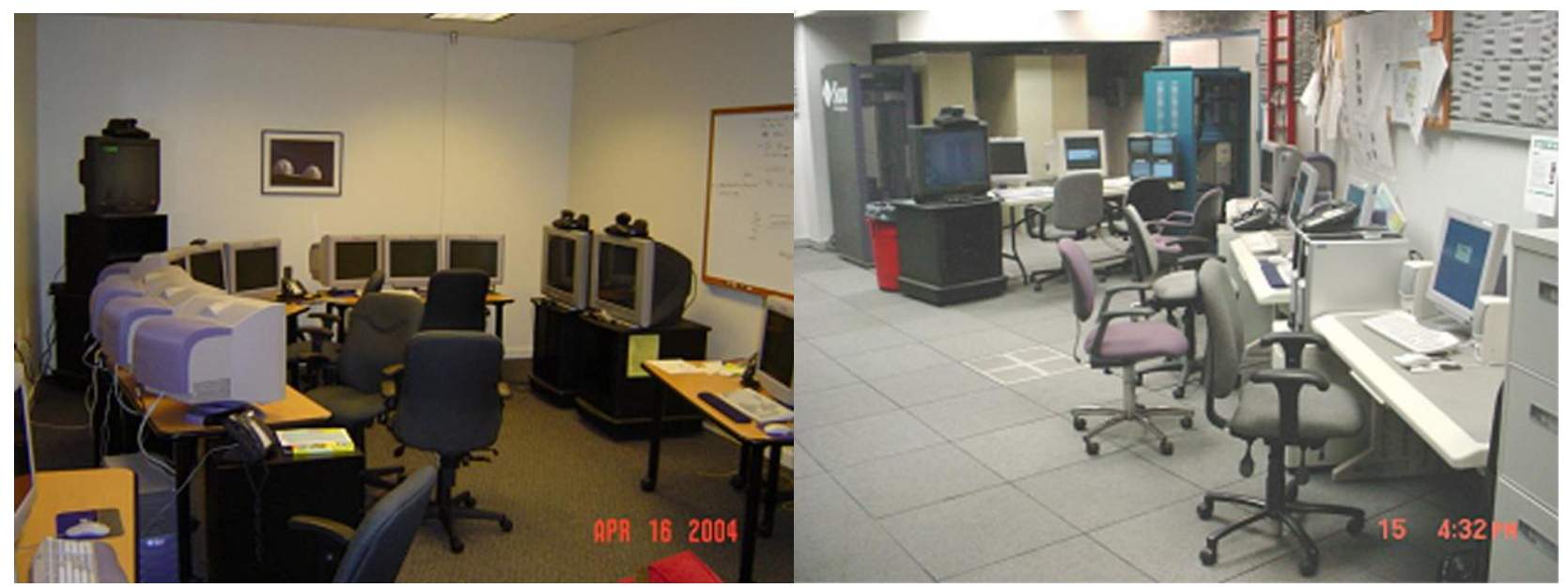

Fig. 9. Headquarters (left) and summit (right) interferometer control rooms. 
The following formal process is associated with each observing run:

1. Complete the items identified in a punch-list from the previous observing run and work on engineering change requests (ECRs).

2. System checkout and alignment begins approximately one week before the run.

3. A pre-run meeting is held approximately one week before the run to review the observing engineering plan, the science plan, the list of observing support personnel, and the status of the punch-list and ECRs.

4. On each day of the run, a pre-observing procedure, including a checklist, is performed.

5. On each day of the run, a 4 p.m. meeting is held to review the instrument status, to identify any remaining daytime tasks, to review the results from the previous night and to review and adjust the night's observing plan.

6. An observing log and a night-log are maintained and distributed. The observing log is a detailed summary of the observations. The night-log records a top-level summary of the observations and details of any problems including time lost. The night-log provides feedback to the rest of the Observatory on any problems that may require resolution.

7. Within a week of the end of the run two post-run meetings are held, one with the interferometer team and one with Observatory and Project management, the old punch-list is closed out and a new punch-list is opened, and a run summary document is distributed.

Operations procedures for daytime checkout and nighttime operation are available on-line at the Web site shown in Figure 10.

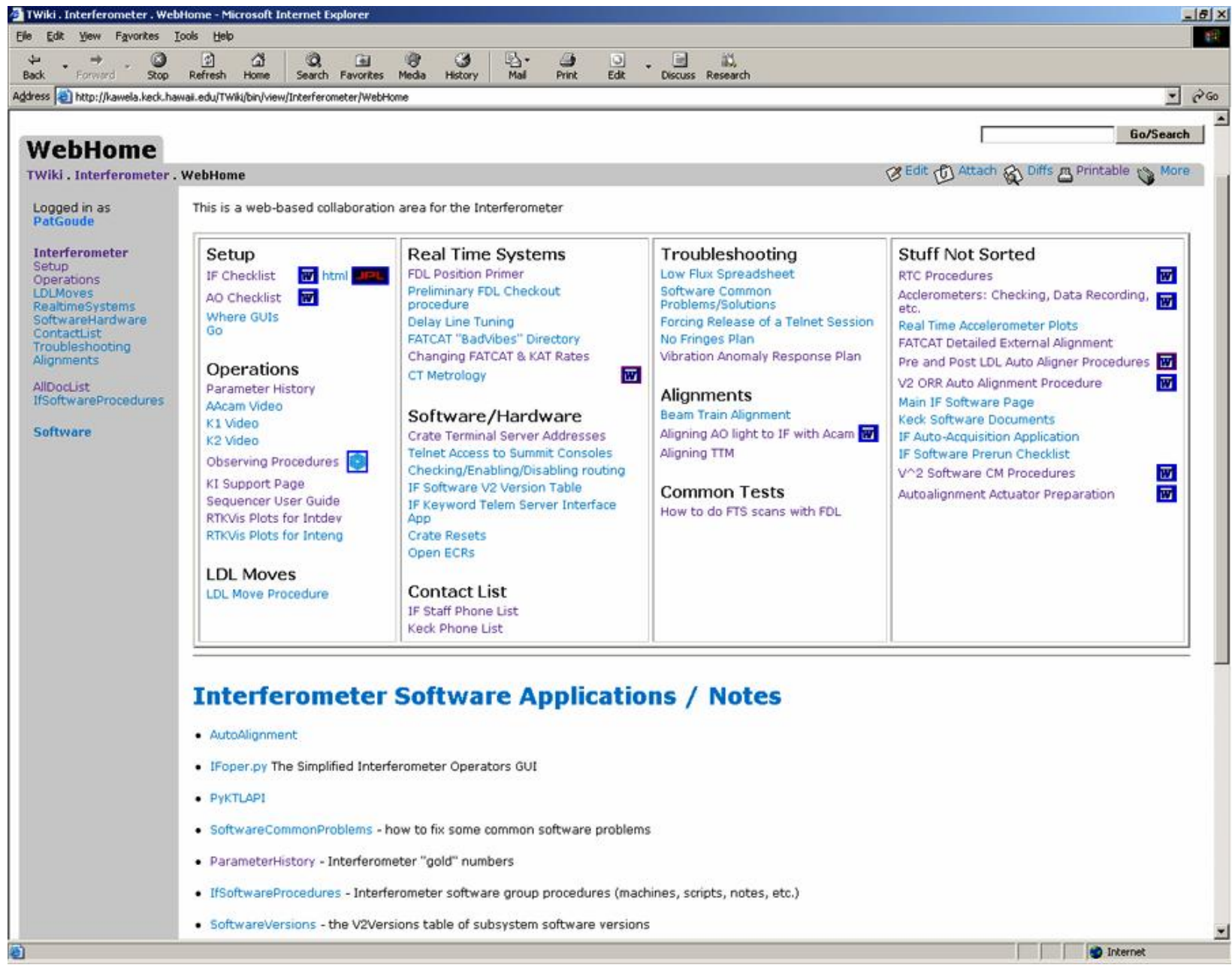

Fig. 10. On-line operations procedures. 
WMKO's standard approach is used for anomaly response:

1. Resolve previously identified problems and/or provide procedures, tools and training for recovery.

2. Identify problems before the start of observing.

3. Provide more on-site assistance early in the night.

4. Resolve problems via training and procedures.

5. Call for assistance soon if a problem is not resolved quickly.

Configuration management is particularly important for the Keck Interferometer since development is ongoing. As subsystems are handed over from development to operations, they pass into WMKO's mechanical and electronics configuration management systems, including document control and an engineering change control process. Software configuration management is especially critical. Separate software operations and development baselines are maintained, and the $\mathrm{V}^{2}$ development baseline is used for a preliminary checkout of changes. A software engineering change control process, including a change control board, has been instituted to document changes and to protect the operational software.

\section{ACKNOWLEDGEMENTS}

The research described in this paper was carried out at the W. M. Keck Observatory, and at the Jet Propulsion Laboratory, California Institute of Technology, under a contract with the National Aeronautics and Space Administration. The Keck Interferometer is funded by the National Aeronautics and Space Administration as part of its Origins Program. The W. M. Keck Observatory is operated as a scientific partnership among the California Institute of Technology, the University of California, and the National Aeronautics and Space Administration. The Observatory was made possible by the generous financial support of the W. M. Keck Foundation.

\section{REFERENCES}

1. R. L. Akeson, "Keck interferometer science: present and future," in New Frontiers in Stellar Interferometry, Proc. SPIE, 5491, 2004.

2. M. R. Swain, "Observing NGC 4151 with the Keck Interferometer," in New Frontiers in Stellar Interferometry, Proc. SPIE, 5491, 2004.

3. M. M. Colavita and P. L. Wizinowich, "Keck interferometer update," in Interferometry for Optical Astronomy II, W. A. Traub, ed., Proc. SPIE, 4838, 79-88, 2003.

4. M. M. Colavita, P. L. Wizinowich, R. L. Akeson, "Keck Interferometer status and plans," in New Frontiers in Stellar Interferometry, Proc. SPIE, 5491, 2004.

5. P. Wizinowich et al., "Adaptive optics developments at Keck Observatory," in Advancements in Adaptive Optics, Proc. SPIE, 5490, 2004.

6. M. Hrynevych et al., "Keck Interferometer autoaligner: algorithms and techniques," in New Frontiers in Stellar Interferometry, Proc. SPIE, 5491, 2004. 